\title{
SUNYAEV-ZEL'DOVICH EFFECT OBSERVATION PROJECT WITH THE NOBEYAMA 45-M TELESCOPE
}

\author{
M. TSUBOI, T. OHNO AND A. MIYAZAKI \\ Ibaraki University, Mito, Ibaraki, 310, Japan \\ T. KASUGA \\ Hosei Univeristy, Koganei, Tokyo, 184, Japan \\ AND \\ A. SAKAMOTO AND T. NOGUCHI \\ Nobeyama Radio Observatory, Nagano, 384-13, Japan
}

\section{Introduction}

The combination of X-ray and Sunyaev-Zel'dovich (S-Z) effect observations toward cluster of galaxies gives the independent estimation of Hubble constant (Sunyaev and Zel'dovich 1970). The measurement of S-Z effect is one of the most difficult observations in radioastronomy because of the weakness of the effect, $\Delta T=0.1-1 \mathrm{mK}$ (e.g. Rephaeli 1995). Because the field of view of the exist interferometers is smaller than the extended distribution of S-Z effect of low redshifted clusters, single-dish telescopes gain an advantage over interferometers. In addition, to reduce the contaminations from Galaxy and galaxies in the cluster, the $\mathrm{mm}$-wave observation is preferable. Thus, we have started the project of S-Z effect observation with the Nobeyama 45-m telescope, which is the largest mm-wave telescope in the world. Our scientific goal is reliable measurement of S-Z effect of many clusters. To realize this we have made a multi-feed PCTJ-SIS mixer receiver at $40 \mathrm{GHz}$ as a sophisticated tool for the observation of S-Z effect (Noguchi et al. 1995, Kasuga et al. 1995, Tsuboi et al. 1997).

\section{Receiver System}

The receiver system consists of 3 dual feeds differential radiometers in order to reject weather and ground effects. Scanning observation can depict 


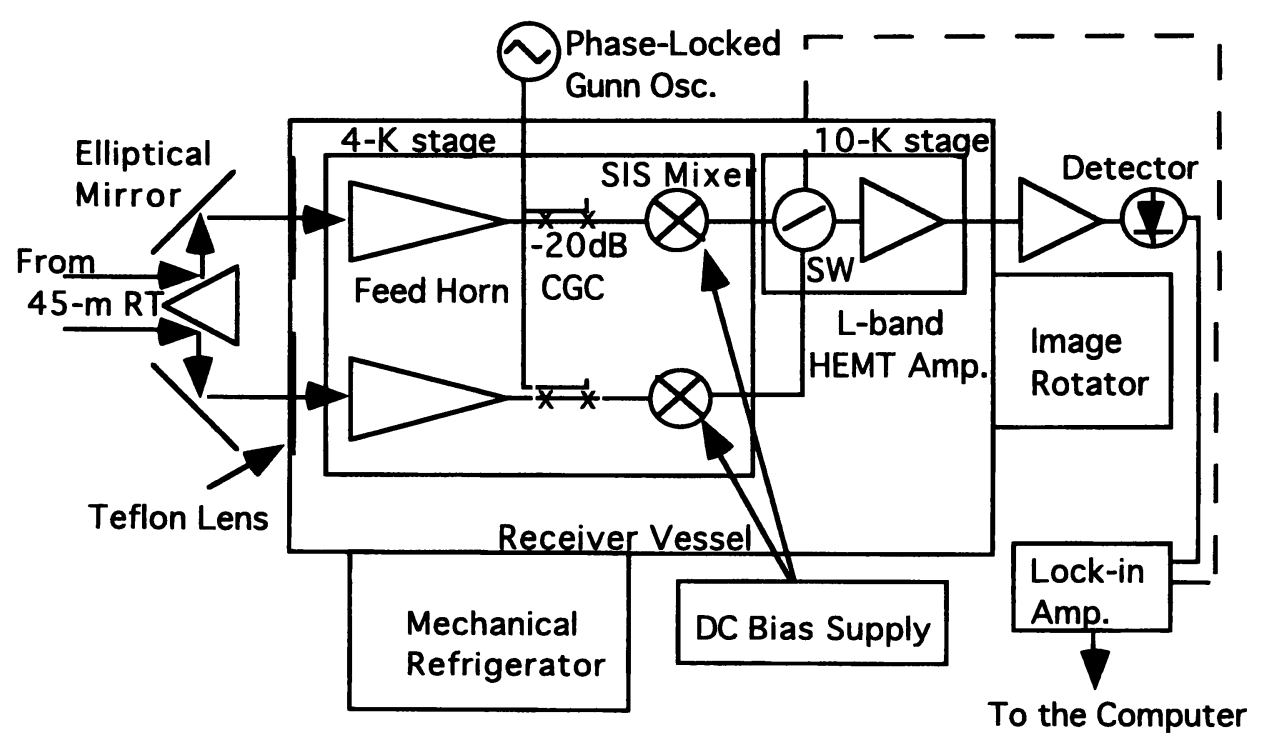

Figure 1. The block diagram of the receiver system

the spatial distribution of S-Z effect. Figure 1 shows the block diagram of the receiver system. The SIS mixers and the horn antennas are located in the Dewar and cooled to $4 \mathrm{~K}$ by a mechanical refrigerator. The low-noise amplifiers are on $15-\mathrm{K}$ stage in the Dewar. The receiver noise temperature is below $60 \mathrm{~K}$ and the instantaneous bandwidth is $700 \mathrm{MHz}$. The beam size is 34 ". The neighboring incident beams are divided by the image dicing mirror on the focal plane. The beams are located at $2 \times 3$ grid with 90 " interval on the sky. The system was installed on the focal plane platform of the 45-m telescope in May 1997. Now, only one beam is available. The beam efficiency and the aperture efficiency are 0.5 and 0.8 , respectively. We are focalizing other beams of the receiver system for the test observation in the winter of 1997-8.

\section{References}

Kasuga, T., Tsuboi, M., Miyazaki, A., Noguchi, T., and Sakamoto, A. (1995) URSI General Assembly

Noguchi, T., Shi, S. -C., and Inatani, J. (1995) IEEE Trans. Appl. Superconductivity, Vol.5, pp 2228-2231

Rephaeli,Y. (1995) Ann Rev. Ann. Astrophys., Vol.33, p541

Sunyaev, R. A. and Zel'dovich, Y. B. (1970) Astrophys. and Space Sci., Vol.7, p3

Tsuboi,M., Miyazaki,A, Kasuga,T.,Sakamoto, A, and Noguchi, T. (1997) TWAA'96, pp99-103 